\section{USING BURDEN OF CANCER TO PROMOTE POLICY CHANGE}

\begin{abstract}
1,2PA Demers* ${ }^{1,3}$ J Kim, ${ }^{1} \mathrm{M}$ Pahwa, ${ }^{4} \mathrm{CE}$ Peters, ${ }^{1} \mathrm{C}$ Song, ${ }^{1,2} \mathrm{VH}$ Arrandale, ${ }^{5} \mathrm{HW}$ Davies, ${ }^{6} \mathrm{~F}$ Labrèche, ${ }^{6,7} \mathrm{~J}$ Lavoue, ${ }^{8} \mathrm{AM}$ Nicol, ${ }^{5} \mathrm{CB}$ McLeod. ${ }^{1}$ Occupational Cancer Research Centre, Cancer Care Ontario, Toronto, Canada; ${ }^{2}$ Dalla Lana School of Public Health, University of Toronto, Toronto, Canada; ${ }^{3}$ Department of Epidemiology, Biostatistics, and Occupational Health, McGill University, Montreal, Canada; ${ }^{4}$ Department of Health Sciences, Carleton University, Ottawa, Canada; ${ }^{5}$ School of Population and Public Health, University of British Columbia, Vancouver, Canada; ${ }^{6}$ School of Public Health, Université de Montréal, Montreal, Quebec, Canada; ' Centre de recherche du Centre hospitalier de I'Université de Montréal, Montreal, Quebec, Canada; ${ }^{8}$ Faculty of Health Sciences, Simon Fraser University, Burnaby, Canada
\end{abstract}

\subsection{6/oemed-2018-ICOHabstracts.417}

Introduction Occupational cancer is poorly recognised due to its long latency among other factors. Burden of occupational cancer projects are an effective tool for drawing attention to the magnitude of the problem and promoting prevention. We present here some major results from the Canadian Burden of Occupational Cancer project and discuss its use for prevention.

Methods Our methods are based on a similar UK project, with several enhancements. The historic labour force was identified using 1961 through 2001 Census data. Occupational exposure prevalence was estimated using CAREX Canada data, allowing calculation of levels of exposure for 44 carcinogens. Relative risks were based on large epidemiologic studies and meta-analyses representative of Canadian exposure circumstances. Standard methods with Levin's equation were used to calculate attributable fractions.

Result Exposure to solar radiation was responsible for the largest number of cancers (4550 basal and squamous cell carcinomas annually, $6.3 \%$ of all non-melanoma skin cancer). Asbestos came second with 425 mesotheliomas $(80.5 \%$ of mesothelioma, including para-occupational, but not environmental, exposure), 1900 lung cancers (8.0\% of lung cancer), and smaller numbers of larynx and, ovarian, and suspected other cancers. Diesel engine exhaust was responsible for 560 lung cancers $(2.4 \%$ of lung cancer) and suspected for 200 bladder cancers $(2.7 \%$ of bladder cancer). Crystalline silica and welding fumes were responsible for 570 and 315 lung cancers $(2.4 \%$ and $1.3 \%$ of all), respectively. Shiftwork resulting in circadian disruption was suspected for between 460 and 1180 breast cancers $(2.0 \%-5.2 \%$ of all), annually.

Discussion Results from the project have begun to be used by Canadian regulatory organisations to establish priorities and by advocacy organisations to raise awareness and push for policy change. These efforts and the input from burden estimates have played a role in the governmental campaign to ban asbestos, now scheduled for 2018.

\section{OCCUPATIONAL HEALTH PROGRAM FOR MIGRANT WORKERS IN THAILAND}

'S Arphorn*, ${ }^{2} \mathrm{P}$ Khongnil, ${ }^{3} \mathrm{G}$ Bangkadanara, ${ }^{1} \mathrm{~T}$ Thanachoksawang, ${ }^{4} \mathrm{~B}$ Wangkahad. ${ }^{1}$ Department of Occupational Health and Safety, Mahidol University, Bangkok, Thailand; ${ }^{2}$ Kanchanaburi College of Agriculture and Technology, Kanchanaburi, Thailand; ${ }^{3}$ School of Health Science, Sukhothai thammathirat Open University, Nonthaburi, Thailand; ${ }^{4}$ Syngenta Crop company, Bangkok, Thailand

10.1136/oemed-2018-ICOHabstracts.418

Introduction Migrant workers play an important role in generating productivity of Thailand presently. The employment of these migrant workers generates social and cultural interaction. Working in agriculture is common among migrant workers especially in border areas. Thailand has an occupational health working mechanism to promote and encourage work safety especially for workers in the system. Examples of this mechanism include the work of safety officers in work safety education, improving working environment, identifying potential hazards, and health check-up based on work risks, etc. These mechanisms are not found in the informal workers, especially among agricultural migrant workers. Apart from the lack of support for occupational health knowledge, there also be communication problems. Use of language to communicate directly to migrant workers may be a problem and an obstacle, sometimes causes misunderstandings.

Objective The aim of this project is to develop a knowledge mechanism driven by the occupational health leader who can understand both Thai and migrant workers' language in order to provide the right and understandable knowledge by learning materials.

Method The researcher has developed a safety promotion model to have the occupational health leader to create work safety characteristics and behaviours as well as transfer this knowledge to migrant workers. The action research collects samples by mixed methodology, applying both qualitative and quantitative research in order to create improvement. The research uses participation technique along with conducting field experiment to test the effectiveness of tools or equipment or patterns or mechanisms developed with the target group.

Result Occupational health leaders can convey the knowledge of the agricultural chemicals risks, and make the migrant workers aware of potential dangers, know how to use selfprotective equipment from agricultural chemicals and have better health.

\section{ERGONOMIC RISK FACTORS, MUSCULOSKELETAL SYMPTOMS, EXERTION AND WORKLOAD AMONG PHYSIOTHERAPISTS IN A SINGLE REHABILITATION CENTRE}

${ }^{1}$ Joshua Samuel Rajkumar*, ${ }^{2}$ Deepak Sharan. ${ }^{1}$ Department of Orthopaedics, RECOUP Neuromusculoskeletal Rehabilitation Centre, Bangalore, India; ${ }^{2}$ Research and Development, RECOUP Neuromusculoskeletal Rehabilitation Centre, Bangalore, India

\subsection{6/oemed-2018-ICOHabstracts.419}

Introduction Physiotherapists (PTs) apply manual forces such a pushing, pulling and lifting, maintain hazardous postures and static loading during treatment, which predisposes them to work related musculoskeletal disorders (WRMSD). Hence, the aim of this study was to evaluate the risk factors for WRMSDs in a group of PTs working in a rehabilitation centre.

Methods A prospective study was conducted among 120 PTs working in a neuromusculoskeletal rehabilitation centre in an Industrially Developing Country. Musculoskeletal and neurological conditions in adult and paediatric populations were primarily treated in the centre. The PT's were evaluated with a self-reported questionnaire which included demographic data, short-form Work Style Questionnaire, Nordic Musculoskeletal Pain Questionnaire, Borg CR 10 and NASA Task Load Index. The inclusion criteria were: PTs working for a minimum of 6 months of experience in the same centre, treating a minimum 
of 6 hours per day and had availed no more than 15 days of leave in the last 6 months.

Result The mean age of the PTs was 29.5 years. On an average, the PTs worked for $8 \pm 1.2$ hours per day for 6 days a week. $78 \%$ of the PTs complained of pain or discomfort within the past 6 months. The commonest sites of pain were lower back (58\%), neck (52\%), upper back (50\%), wrist and hand $(35 \%)$, shoulder (32\%) and ankle (10\%). Analysis of short form of workstyle questionnaire revealed that $70 \%$ of the subjects reported an adverse workstyle risk (total score $>28$ ). The perceived exertion and workload were also high as over $75 \%$ of the PTs had scores of $>15$ (Borg CR 10) and $>50$ (NASA Task Load Index) respectively.

Discussion PTs handling both adult and paediatric patients had high risk of developing WRMSD and appropriate recommendations were given based on the results to ensure prevention of WRMSD.

\section{EFFECTIVENESS OF ONSITE OCCUPATIONAL HEALTH CLINICS IN MANAGEMENT OF WORK RELATED MUSCULOSKELETAL DISORDERS IN 10,850 INFORMATION TECHNOLOGY PROFESSIONALS}

\begin{abstract}
${ }^{1}$ Deepak Sharan*, ${ }^{2}$ Joshua Samuel Rajkumar, ${ }^{2}$ Rajarajeshwari Balakrishnan, ${ }^{3}$ Jerrish A Jose. ${ }^{1}$ Department of Orthopaedics, RECOUP Neuromusculoskeletal Rehabilitation Centre, Bangalore, India; ${ }^{2}$ Research and Development, RECOUP Neuromusculoskeletal Rehabilitation Centre, Bangalore, India; ${ }^{3}$ Corporate Services, RECOUP Neuromusculoskeletal Rehabilitation Centre, Bangalore, India
\end{abstract}

\subsection{6/oemed-2018-ICOHabstracts.420}

Introduction Onsite clinics play a crucial role in the provision of occupational health services. However, the effectiveness of onsite clinics in the management of work related musculoskeletal disorders (WRMSD) is an under studied area. This study reports the outcome of onsite occupational health clinics in Information Technology (IT) companies in the management of WRMSD over a 10 year period.

Methods Aprospective study was conducted from 2006 to 2016, covering 10850 employees of IT companies in different cities in an Industrially Developing Country. The employees (6990 males and 3860 females, between the ages 20 to 60 years), were diagnosed by an experienced occupational health physician (OHP) to have a WRMSD in specific regionsfollowing extensive usage of desktop and/or laptop computer. All the employees then underwent an ergonomic workplace analysis and protocol based rehabilitation for the WRMSD by specially trained occupational physiotherapists. The employees were reviewed by the OHP monthly and at the completion of rehabilitation.

Result Most employees were software and application engineers, followed by managers and technical support staff. A total of $62 \%$ of the employees worked for at least 5-9 hours per day and 38\% for 10-14 hours per day. The predominant symptoms were low back pain (for males) andneck pain (for females). Nearly, 55\% were diagnosed to haveMyofascial Pain Syndrome and others with Thoracic Outlet Syndrome, Fibromyalgia, Tendinopathies and Type 1 Complex Regional Pain Syndrome. After the rehabilitation, the VAS scale showed significant reduction in pain levels $(\mathrm{p}<0.01) .78 \%$ had reported reduced productivity due to the WRMSD, which improved markedly after the rehabilitation. 93\% of workers reported complete resolution of symptoms and $7 \%$ reported partial resolution of symptoms but could work without restriction.
Discussion Onsite occupational health clinics are effective in the managementof WRMSD in IT companies.

\section{WORK RELATED MUSCULOSKELETAL DISORDERS AMONG ORTHOPAEDIC SURGEONS: A SURVEY STUDY}

${ }^{1}$ Deepak Sharan*, ${ }^{2}$ Joshua Samuel Rajkumar, ${ }^{2}$ Rajarajeshwari Balakrishnan. ${ }^{1}$ Department of Orthopaedics, RECOUP Neuromusculoskeletal Rehabilitation Centre, Bangalore, India; ${ }^{2}$ Research and Development, RECOUP Neuromusculoskeletal Rehabilitation Centre, Bangalore, India

\subsection{6/oemed-2018-ICOHabstracts.421}

Introduction Surgeons, especially Orthopaedic Surgeons (OS) maintain awkward postures and repetitive tasks which are ergonomically risky. However, there is a paucity of data on the prevalence of work related musculoskeletal disorders (WRMSD) among OS. Hence, the objective was to evaluate the prevalence and risk factors of WRMSD among OS.

Methods A survey was conducted using a structured questionnaire, disseminated online. There were 57 respondents, who were OS with a minimum working experience of one year, and the surgeons were practicing in the field of orthopaedics only. The structured questionnaire included demographic details such as age, sex, height, weight, total work experience, number of working hours in a day, type or department of work, questions related to regular exercise, physical risk factors associated with working condition, present health status. Nordic Musculoskeletal Questionnaire (NMQ) was used to know the regional involvement, prevalence and disability rate of MSD, in past 7 days or during the last 12 months. The short form of work style questionnaire was used to assess the risk factors of adverse work style. Data were recorded and analysed.

Result The mean age of the OSs was 46.32 years and were predominantly males (96\%). On an average, the percentage of OSs with operating hours more than 14 hours was $75.4 \%$. Joint replacement (50.9\%) and Arthroscopic surgeries (35.1\%), were the commonest surgical procedures carried out by them. A high prevalence rate of work related musculoskeletal symptoms among OS was found, mainly in the low back (68.42\%), neck (56.14\%), shoulder (42.1\%) and upper back (31.57\%) regions. Sustained static and/or awkward posture was perceived as the factor most commonly associated with low back and neck symptoms by $84.2 \%$ of respondents.

Discussion A high prevalence of musculoskeletal symptoms was reported among OS and interventions to address the risk factors identified are recommended.

\section{RISK FACTORS FOR THE DEVELOPMENT OF WORK RELATED MUSCULOSKELETAL DISORDERS AMONG INFORMATION TECHNOLOGY PROFESSIONALS}

'Deepak Sharan*, ${ }^{2} J$ oshua Samuel Rajkumar. 'Department of Orthopaedics, RECOUP Neuromusculoskeletal Rehabilitation Centre, Bangalore, India; ${ }^{2}$ Research and Development, RECOUP Neuromusculoskeletal Rehabilitation Centre, Bangalore, India

10.1136/oemed-2018-ICOHabstracts.422

Introduction Work Related Musculoskeletal Disorders (WRMSD) are highly prevalent among Information Technology (IT) professionals. However, the risk factors associated with the development of WRMSD are not clear. Hence, the 